\title{
Diálogo de saberes: justicia indígena del Cauca y justicia ordinaria, un intercambio cultural posible
}

\author{
Dialogue of knowledge: indigenous justice in Cauca and \\ ordinary justice, a possible cultural exchange \\ Diálogo do conhecimento: justiça indígena Cauca e justiça \\ ordinária, um possível intercâmbio cultural \\ Epu rume kimün güxam: Pu ka mapuche ñi norüm zugu \\ kimün Cauca chegelu ka ka xipache ñi norüm zugun, \\ ñi feygeal chi welulgeal mogen kimün zugu
}

Jaime Antonio Álvarez Soler ${ }^{1}$ Universidad del Cauca, Colombia

Pero se llegará ese día en que la legislación indígena por ella misma será encaminada rápidamente a formar su tribunal y destruirá la envidia y el error que ejecutaron a sabiendas y con conocimiento de causa los señores aristocráticos, que sin justicia y sin caridad nos han hecho desterrar por medio de leyes subversivas [...]. Manuel Quintín Lame ${ }^{2}$

Recepción: 26/03/2019

Evaluación: 15/05/2019

Aceptación: 23/05/2019

Artículo de Investigación

DOI: https://doi.org/10.19053/01227238.9097

\section{RESUMEN}

Dentro de la investigación llevada a cabo para mi tesis doctoral titulada "Fenomenología de la educación médica en la Universidad del Cauca", se evidenció la ausencia de una relación entre las co-

munidades indígenas y el programa de Medicina de dicha universidad, lo cual motivó el desarrollo de un proyecto de investigación-acción con los pueblos indígenas del departamento del Cauca.

1 Médico, patólogo, profesor asociado Universidad del Cauca, Departamento de Patología, Grupo de Investigación Educación Rural, UPTC, estudiante Doctorado en Ciencias de la Educación -RUDECOLOMBIA-UPTC correo elctronico: jalvarezs@unicauca.edu. co.

2 "El derecho de la mujer indígena en Colombia", en Documentos para la historia del movimiento indígena colombiano contemporáneo, comps. Enrique Sánchez y Hernán Molina (Bogotá: MinCultura, 2010), 32. 
En la aplicación de una "justicia propia" ejercida ancestralmente - sin la intervención directa del Estado-por los pueblos indígenas del Cauca, sus autoridades han detectado situaciones de juzgamientos inadecuados, muchos de ellos injustos, y con serios vacíos probatorios dentro de los procesos. Teniendo en cuenta esta realidad, el texto sobre el cual se fundamentó este artículo tuvo como punto de partida las siguientes preguntas: ¿cómo se ejerce la práctica de la "justicia propia" de los pueblos indígenas del Cauca?, y ¿qué estrategias pedagógicas participativas permiten la interacción y recreación de saberes entre los pueblos indígenas, el Instituto Nacional de Medicina Legal y Ciencias Forenses (INMLCF) y la Universidad del Cauca, como parte del constructo intercultural para la protección de los Derechos Humanos de todas las personas? Es así como se traza un objetivo orientado a dar sentido académico a las preguntas planteadas: construir en forma conjunta - autoridades indígenas, INMLCF y la Universidad del Caucaun diseño curricular para capacitar en procedimientos médicos forenses y disección de cadáveres a la guardia indígena y demás autoridades de apoyo. El proceso pedagógico y las prácticas interculturales permitieron el intercambio de saberes, pero, sobre todo, una aproximación a la comprensión y cosmovisón de la "justicia indígena". Fue una experiencia significativa que generó nuevos conceptos útiles para entender la "justicia propia" de los pueblos indígenas y los aportes negociados que la justicia ordinaria colombiana puede ofrecer a esta práctica ancestral.

Palabras clave: Investigación social; educación intercultural; identidad cultural.

\section{ABSTRACT}

In my doctoral research work, entitled "Phenomenology of medical education at the Universidad del Cauca", the absence of a relationship between the indigenous communities and the Medicine Program of this university was found. This fact motivated the creation of a research-action project with the indigenous peoples of the department of Cauca.

In the application of an ancestral system of "own justice" exercised - without the direct intervention of the State - by the indigenous peoples of Cauca, their authorities have detected situations of inadequate judgments, often unfair, and with serious evidentiary gaps within the processes. Bearing this reality in mind, the base text of this paper proposed the following questions: How is the "own justice system" applied by the indigenous peoples of Cauca?, and what participatory pedagogical strategies allow for the interaction and recreation of knowledge among indigenous peoples, the Instituto Nacional de Medicina Legal y Ciencias
Forenses (National Legal Medicine Institute) and the Universidad del Cauca, as part of the intercultural construct for the protection of the human rights of all people? In this way, an objective was set to make academic sense of the questions posed: to construct, together with the three parties involved, a curricular design to train the indigenous guard and other official support authorities in forensic procedures and dissection of corpses.

The pedagogical process and the intercultural practices allowed the exchange of knowledge, but in particular, an approach to the understanding and cosmovison of the "indigenous justice". It was a significant experience that generated new useful concepts to understand the "own justice system" of the indigenous peoples and the negotiated contributions that the Colombian ordinary justice can offer to this ancestral practice.

Key words: Social research; intercultural education; cultural identity. 


\section{RESUMO}

Durante a pesquisa desenvolvida na tese doutoral intitulada "Fenomenologia do ensino médico na Universidade do Cauca", evidenciou-se a ausência de relação entre as comunidades indígenas do estado do Cauca e o programa de Medicina da referida universidade, o que incentivou a realização de um projeto de pesquisa-ação em conjunto com os povos indígenas do estado de Cauca. Ao analisar como os povos indigenas aplicam uma "justiça própria" exercida sem a intervenção direta do Estado, atividade que faz parte do seu comportado ancestral, as autoridades detectaram situações de julgamentos inadequados, em muitas ocasiões injustos, alem de evidenciar lacunas probatórias graves nos processos. Diante dessa realidade, o texto em que este artigo foi baseado teve como ponto de partida as seguintes perguntas: Como é a prática da "justiça própria" exercida pelos povos indígenas do estado do Cauca?, e, Quais estratégias participativas pedagógicas permitem a interação e a recriação do conhecimento entre os povos indígenas, o Instituto Nacional de Medicina Legal e Ciências Forenses (INMLCF) e a Universidade do Cauca, como parte do construto intercultural para a proteção dos Direitos Humanos de todas as pessoas? Assim, se construiu um objetivo orientado a fornecer sentido acadêmico às questões colocadas, a saber: "Construir conjuntamente entre povos indígenas, o INMLCF e a Universidade do Cauca um desenho curricular para treinar em procedimentos médicos forenses e dissecação de cadáveres à guarda indígena e a outras autoridades de apoio dos povos indígenas". A abordagem metodológica baseou-se nas Pedagogias de Pesquisa-Ação Participativa (IAP). O processo pedagógico e a ação das práticas interculturais possibilitaram a troca de conhecimentos, e primcipalmente, permitiram uma abordagem de compreensão e cosmovisão sobre as práticas de "justiça indígena". A execução deste projeto foi uma experiência significativa, na que a troca de conhecimento entre diferentes culturas gerou novos conceitos úteis para entender a "justiça própria" dos povos indígenas e as contribuições negociadas que a justiça ordinária colombiana pode oferecer a essa prática ancestral.

Palavras-chave: Pesquisa social; educação intercultural; identidade cultural

\section{PIKÜNOGETUN}

Zewmanmu ta ñi tesis doctoral "Fenomenología de la educación médica en la Universidad del Cauca" pigelu, kimgey ñi mülenon kiñe xaftuwün pu comunidades indígenas egu programa de Medicina fey chi füxa chijkatuwe ruka mew, femgechi ta rakizuamgey nentual kiñe küzaw investigación-acción tüfa chi pu indígenas egün mülelu departamento Cauca mew.

$\mathrm{Pu}$ indígenas mülelu Cauca mapu mew kuyfi mew ta nentukefuy "kizu ñi wichan" egün -fey mew koneltukelay ta Estado- fey mew ta ñi pu logkolelu güneltuygün ñi xipaken welulkan wichan, tañi wichagenoafel rume, nienon mew zugu ñi zajuntukugeal egün. Penien mew ta tüfa chi zugu, chijkantukugey ta zugu kiñeke ramtun mew: ¿chumgechi am ta xipakey "kizu wichan" pu indígenas Cauca mapu mew?, ka ¿chem pepilkan zugu kümeafuy tañi zugual kom che ka ñi feypial ñi kimün egün, tüfey chi Instituto Nacional de Medicina Legal y Ciencias Forenses (INMLCF) ka fey chi Universidad del Cauca, müleal ta epu rume chi kimün zapinieal ta Derechos Humanos kom pu che mew? Femgechi ta azkünugey kiñe zitul zugu nieal falin chijka zugu ñi ramtun mew: kiñen xür zewmayal -pu indígenas logkolelu, INMLCF ka Universidad del Cauca- kiñe azkünun zugu ñi kimeltugeael egün ñi chumgechi kimcheal bale ta che ka kim malüal ta ba pu indígenas kamañ ka kom logkolelu kejun zugu 
mew. Kimeltun mew ka nentun ta küzaw epu rume kimün mew xipay ta epuñpüle kimuwün, welu zoy fülgey ajkütuwün zugu mew ka kimgey indígenas de mogen ñi chumkünukey ñi "kizu wichan". Kiñe küme zugu nentugey chew ta xipay weke hemül ñi zoy küme kimgeal ta pu indígenas ñi "kizu wichan” ka chumgechi ta justicia ordinaria colombiana faliltuafuy ta kuyfi femken ta che.

Zichul zugun: inatuzugun xokiñ che mew, ерu rume kimeltuwün, che ñi femgen kiñe xokiñ che mew.

\section{INTRODUCCIÓN}

La justicia ordinaria colombiana tiene como base de su ejercicio hacer cumplir los derechos fundamentales, sociales, culturales, políticos y económicos, aspectos todos que han sido contemplados y reformados historicamente desde la Constitución de 1886. La Constitución de 1991 consagró los derechos materiales de los indígenas y además creó algunos instrumentos jurídicos procesales para su defensa. El punto de referencia es su artículo 7: “El Estado reconoce y protege la diversidad étnica y cultural de la Nación colombiana".

Bajo el establecimiento de este mandato de la Constitución Política de Colombia de $1991(\mathrm{CP})^{3}$, se promueve un estatus especial para los pueblos indígenas cuya manifestación se origina en el ejercicio de los derechos de legislación y jurisprudencia dentro de su área territorial, en concordancia con sus propios valores y cultura (artículo 246), mediante la autogestión de poderes propios dentro de sus usos y constumbres (artículo 330) y el ejercicio irrestricto de la propiedad sobre sus resguardos y territorios ${ }^{4}$.

Aunque el hecho de que las leyes colombianas no hubieran reconocido a la "justicia propia" como pieza fundamental, no quiere decir que su práctica ancestral desapareciera, dado que la $\mathrm{CP}$, al reconocer la diversidad cultural, deja claro que respeta su aplicación; sin embargo, la justicia ordinaria colombiana mantiene un desacuerdo frente a la práctica ancestral de transmisión oral de las tradiciones de los pueblos indígenas. A pesar de que subsisten estos desencuentros entre la "justicia propia" indígena y la justicia ordinaria, existen acercamientos, "donde se debe seguir aprendiendo a partir de respetar las diferencias entre cada una" 5 .

En un proceso de acercamiento entre la "justicia propia" y la justicia ordinaria, y teniendo en cuenta algunas desaveniencias en casos de juzgamiento indígena, el Consejo Regional Indígena del Cauca (CRIC) solicitó al Instituto Nacional de Medicina Legal y Ciencias Forenses (INMLCF), su colaboración en procesos de capacitación científica para hacer una justicia adecuada a quienes cometen delitos en sus comunidades.

3 En adelante la sigla CP hará referencia a la Constitución de 1991.

4 Frank Semper, "Los derechos de los pueblos indígenas de Colombia en la jurisprudencia de la Corte Constitucional", Anuario del Derecho Constitucional Latinoamericano (2006): 764, http://www.corteidh.or.cr/tablas/R21731.pdf.

5 "Encuentros y desencuentros entre la Justicia propia y la justicia ordinaria”, Consejo Regional Indígena del Cauca - CRIC, 2018, acceso el 7 de junio de 2019, https://www.cric-colombia.org/portal/encuentros-desencuentros-la-justicia-la-justicia-ordinaria/. 
Teniendo en cuenta esta situación, y con el objetivo de comprender las prácticas de estos pueblos indígenas en el tema, además de poder estructurar una propuesta de formación de carácter participativa, se partió de las siguientes preguntas: ¿cómo se ejerce la "justicia propia" de los pueblos indígenas del Cauca?, ¿qué estrategias pedagógicas participativas permiten la interacción y recreación de saberes entre los pueblos indígenas, el INMLCF y la Universidad del Cauca? Con base en estos cuestionamientos se trazó el siguiente objetivo general: establecer un diálogo de saberes que permitiera reconocer las prácticas en la aplicación de la justicia propia de los pueblos indígenas del Cauca y diseñar y ejecutar estrategias pedagógicas participativas que condujeran a la interacción y recreación de saberes entre los pueblos indígenas, el INMLCF y la Universidad del Cauca, como parte del constructo de una justicia intercultural.

En este orden de ideas, los objertivos específicos estuvieron orientados a promover el intercambio de nuevos conceptos, saberes y procedimientos de la justicia indígena, desconocidos por la justicia ordinaria, el INMLCF y la Unicauca; diseñar un currículo para disectores forenses y asistentes en procedimientos forenses, que solucionara las necesidades de los pueblos y de la Guardia Indígena, especialmente en la realización de procedimientos técnicos forenses como autopsias y levantamientos de cadáveres; gracias a estas acciones, por primera vez una mujer indígena se recibió como asistente en técnicas y procedimientos forenses.

El abordaje metodológico se fundamentó en la IAP, planteada por Fals Borda ${ }^{6}$ a partir del diálogo de saberes y la observación participante como técnicas que favorecen el intercambio cultural, elimina las hegemonías y facilita la construcción de nuevo conocimiento, donde interactúan diversas miradas y cosmovisiones. El proceso se desarrolló a partir de la metodología de acercamiento con los pueblos indígenas del CRIC, la formación de mesas de discusión y talleres, con un diagnóstico posterior y co-construcción de un plan de acción planificado y la ejecución de acciones concertadas entre los pueblos indígenas y las entidades participantes.

\section{Indígenas del Cauca (Colombia)}

Politica PEl departamento del Cauca está ubicado en el sur occidente de Colombia, entre las regiones Andina y Pacífica. Tiene una superficie de 29308 kilómetros cuadrados y se encuentra dividido en 38 municipios. Según el Departamento Nacional de Estadística (DANE), en 2017 la población del departamento estaba estimada en 1404205 personas, de las cuales mestizos y blancos conformaban el 53,3 \%, negros o afrocolombianos el 22,3\% e indígenas el 21,6\%, porcentaje este último que corresponde a una población de 303308 habitantes $^{7}$.

6 Orlando Fals Borda y Carlos Rodrigues Brandão, Investigación participativa (Montevideo: Instituto del Hombre/Ediciones de la Banda Oriental, 1986).

7 Departamento Administrativo Nacional de Estadística (DANE), acceso el 25 de marzo de 2019, https://www.dane.gov.co/ 
Según el CRIC, en el departamento del Cauca se encuentran los siguientes pueblos indígenas: Nasa, Eperara Siapidara, Kishu-kisgó, Ambaló, Totorótontotuna, Polidara, Misak, Kokonuko, Yanacona, Inga, Pubenese, pueblos que se han organizado y declarado históricamente en resistencia debido a los múltiples hechos violentos de los que han sido víctimas. Estos pueblos están organizados en 84 resguardos, 115 cabildos y 11 asociaciones de cabildo, distribuidos por todo el departamento, predominantemente en el nor-oriente caucano.

Gómez Valencia expresa que las justicias indígenas no son iguales, si no múltiples y diversas ${ }^{8}$. En este sentido, Joe Sauca plantea que, "históricamente, las justicias indígenas han sido influenciadas por las corrientes de la justicia occidentalizada ordinaria, lo que ha llevado al desconocimiento y exclusión de la justicia propia" ${ }^{\prime \prime}$.

El 24 de febrero de 1971, en el municipio de Toribío, Cauca, se organizó el Consejo Regional Indígena del Cauca (CRIC), conformado por siete cabildos e igual número de resguardos indígenas, y se nombró el primer comité ejecutivo. Debido a la represión de los terratenientes de la zona y la poca organización de las comunidades, no fue posible hacerlo funcionar de manera adecuada ${ }^{10}$. En este mismo sentido lo expresa Flórez Schneider:

Hombres y mujeres cansados del despojo de sus tierras y propiedades, de ser llamados "indios cochinos" por los colonos mestizos y ser esclavos en su propia tierra, se unieron para recuperar la madre tierra arrebatada por los terratenientes que los acorralaron hacia estas montañas áridas. Fue en el seno del CRIC donde surgió la Guardia Indígena, como respuesta de protección a sus territorios, identidad y cultura contra las amenazas y violaciones a los derechos humanos y el derecho internacional humanitario por parte de la guerrilla, los paramilitares, la fuerza pública y el narcotráfico que invadió de cultivos de coca esta región ${ }^{11}$.

En Colombia, desde 1991, se reconocen constitucionalmente los derechos de las comunidades indígenas, en su cosmovisión y prácticas autónomas como minorías étnicas y culturales, tal como lo afirma el artículo 7 de la CP: "El Estado reconoce y protege la diversidad étnica y cultural de la Nación colombiana"; es así como queda plasmado en la Carta Magna.

Joe Sauca expresó que, en septiembre de 1971, se realizó el segundo Congreso del CRIC en el municipio de Tacueyó ${ }^{12}$. Allí se definieron los puntos del programa político, cuyas exigencias constituyeron el eje del movimiento y se retomaron enseñanzas de líderes como la Gaitana, Juan Tama de la Estrella y Manuel

\footnotetext{
$8 \quad$ Herinaldy Gómez Valencia, "Justicias indígenas de Colombia: reflexiones para un debate cultural, jurídico y político. Pueblos Kogui, Arhuaco, Wiwa, Kankuamo, Nasa, Misak, Yanacona y Camëntŝa”, Centro de Documentación Judicial (2015), https://www.ramajudicial.gov.co/documents/4263275/0/LibroJusticiasIndigenas.pdf/e932af27-6ca8-462a-8f79-73cd14168426.

9 Joe Sauca (asesor jurídico en derechos humanos del CRIC), en conversación con el autor, Popayán, 21 de mayo de 2018.

10 "Encuentros y desencuentros".

11 Gloria Flórez Schneider, "Cuenten con nosotros para la Paz y NO para la Guerra”, Parlamento Andino, acceso el 4 de junio de 2019 , http://www.oidhaco.org/uploaded/content/article/1499238702.pdf.

12 Sauca, conversación.
} 
Quintín Lame. Con estos criterios las comunidades indígenas fortalecieron las luchas con el fin de lograr la aplicación de la Ley 89 de 1890, donde se planteaba en el artículo 1. : "La legislación general de la República no regirá entre los salvajes que vayan reduciéndose á la vida civilizada por medio de Misiones. En consecuencia, el Gobierno, de acuerdo con la Autoridad eclesiástica, determinará la manera como esas incipientes sociedades deban ser gobernadas". Y en el artículo 3.: "En todos los lugares en que se encuentre establecida una parcialidad de indígenas habrá un pequeño Cabildo nombrado por estos conforme a sus costumbres".

\section{Justicias indígenas}

Es de anotar que la $\mathrm{CP}$ reconoce la multiculturalidad y las leyes propias indígenas. De acuerdo con la exmagistrada Lucía Arbeláez:

Los derechos, el territorio, la organización social, las instituciones de control social y territorial y en general los sistemas judiciales de los indígenas, existen desde tiempos inmemorables heredados de los dioses y de la ley de origen de cada pueblo. Desde luego, eso no significa que los sistemas judiciales y en general las comunidades indígenas, sean estáticos, su diaria vivencia y las nuevas situaciones a las que se enfrentan, dentro de sus comunidades y en relación con la sociedad no indígena, los hace crear y recrear permanente, pero ordenadamente sus sistemas propios, $e$ incluso apropiar elementos que pueden ser útiles a la pervivencia de cada uno de los pueblos ${ }^{13}$.

De manera que, según Esther Sánchez, mediante la Constitución también se definió: “[...] que ellos [las comunidades indígenas] pueden utilizar, como colombianos, además de su propia jurisdicción indígena, la jurisdicción ordinaria cuando se encuentren con conflictos que a su saber y entender entren en las posibilidades de resolución que el marco de esta jurisdicción ofrece, si con ello no contravienen los marcos de competencia que habrán de respetarse"14.

En este orden de ideas, asimismo es necesario reconocer que la CP contempla, en el artículo 246, que: “Las autoridades de los pueblos indígenas podrán ejercer funciones jurisdiccionales dentro de su ámbito territorial, de conformidad con sus propias normas y procedimientos, siempre que no sean contrarios a la Constitución y leyes de la República. La ley establecerá las formas de coordinación de esta jurisdicción especial con el sistema judicial nacional". Es así como se marca la diferencia entre lo planteado en las constituciones de 1886 y 1991; esta última determina un amplio reconocimiento a las comunidades indígenas.

13 Lucía Arbeláez de Tobón, "La Jurisdicción Especial Indígena en Colombia y los mecanismos de coordinación con el Sistema Judicial Nacional”, República de Colombia, Rama Judicial del Poder Público (Guatemala: Consejo Superior de la Judicatura, 2004), 6, acceso el 4 de junio de 2019, https://www.cejamericas.org/Documentos/DocumentosIDRC/21LucaArbelaez.pdf.

14 Esther Sánchez, Derechos propios, ejercicio legal de la Jurisdicción Especial Indígena en Colombia, citada por Lucía Arbeláez en ibíd., 5 . 
Sin embargo, de acuerdo con la Organización Nacional Indígena (ONIC), "[...] en el territorio nacional habitan 102 pueblos indígenas, algunos de ellos reconocidos por algunas instituciones del Estado colombiano [...], pero no por todas ellas". Según el Censo de 2005 solamente se reportan 87 pueblos. Si bien se busca con la norma respetar sus derechos, formas de conviencia, aplicación de justicia y muchas otras facetas de su dinámica y vida organizacional y política, las acciones y la teoría no son coherentes con los retos y el desafío que implica concretarlos en la realidad.

En el artículo 5 de la "Declaración de las Naciones Unidas sobre los derechos de los pueblos indígenas"15 se establece que "Los pueblos indígenas tienen derecho a conservar y reforzar sus propias instituciones políticas, jurídicas, económicas, sociales y culturales, manteniendo a la vez su derecho a participar plenamente, si lo desean, en la vida política, económica, social y cultural del Estado"16.

Para comprender las prácticas de la justicia propia indígena, se acudió a entrevistas con miembros y líderes de los pueblos indígena del Cauca como Joe Sauca y Fernando Caso Chate ${ }^{17}$, quienes expresaron: "La cosmovisión del indígena caucano está basada en la armonía del territorio, en donde la tierra, los animales, las plantas y el hombre, con todos sus recursos naturales, conviven de forma armónica y es allí donde precisamente el hombre, a través de sus comportamientos, acciones y prácticas, genera desarmonización. Esta desarmonización requiere de la intervención de la autoridad indígena en el territorio para rearmonizar, convirtiéndose en un ciclo de armonización, desarmonización y rearmonización".

Según lo planteado por Sauca, este ciclo se encuentra regido por una serie de normas y reglas propias denominadas por ellos como "mandatos", los cuales deben ser cumplidos por toda la comunidad y son regulados y vigilados por los mismos "comuneros" y la autoridad indígena. Esta última tiene un poder de tipo espiritual, mágico y religioso, en donde los médicos tradicionales son quienes le dan soporte. La guardia indígena ejerce funciones como un brazo fuerte en la aplicación de la "justicia propia" en los territorios.

La Asamblea Mayor es el máximo órgano de decisión y juzgamiento de los diferentes pueblos que conforman el CRIC. En este escenario las partes intervinientes: el acusado, la defensa y la autoridad indígena, exponen sus argumentos para el esclarecimiento de las desarmonizaciones, la toma de decisiones y las respectivas condenas y rearmonizaciones. Estas decisiones son tomadas teniendo en cuenta distintas evidencias o elementos probatorios, entre los que pueden estar los dictámenes del INMLCF, institución que realiza estos dictámenes como parte de una obligatoriedad expresada en los acuerdos de cooperación de la Co-

15 En adelante la "Declaración".

16 Naciones Unidas, "Declaración de las Naciones Unidas sobre los derechos de los pueblos indígenas", 107.a sesión plenaria (13 de septiembre de 2007), 4, acceso el 25 de marzo de 2019, https://www.un.org/development/desa/indigenous-peoples-es/declaracion-sobre-los-derechos-de-los-pueblos-indigenas.html.

17 Fernando Caso Chate (consejero mayor del CRIC), en conversación con el autor, Popayán, 21 de mayo de 2018. 
misión Nacional de Coordinación del Sistema Judicial Nacional y la Jurisdicción Especial Indígena (COCOIN) ${ }^{18}$ (https://www.ramajudicial.gov.co/web/jurisdiccion-especial-indigena-y-el-sistema-judicial-nacional/acuerdos-y-compromisos. 2019). Dentro del proceso descrito quedan soportes y actas de juzgamiento, las cuales incluyen las decisiones que toma este órgano colectivo y garantiza su cumplimiento, seguimiento y transparencia.

De acuerdo con José Albeiro Camayo ${ }^{19}$ y Caso Chate, la Guardia Indígena del Cauca tiene como función defender el "Plan de Vida" y mantener la paz y la convivencia en los territorios. Dentro de sus funciones está la vigilancia, control, alarma, protección y defensa de su tierra bajo el mando y coordinacion de la comunidad en defensa de la autonomía.

Los "mandatos" y "Justicia propia" están basados en la "Ley de Origen", el ejercicio del derecho propio y la Constitución Política, artículos 7, 330 y 246.

La Guardia Indígena es la encargada de mantener el "derecho propio" y deriva de los mandatos dados por las asambleas. El símbolo de la guardia se llama "chonta" o bastón, el cual es utilizado para la defensa y es simbólico y les brinda estatus $^{20}$.

La Guardia también realiza labores como búsqueda de personas desaparecidas o secuestradas, primeros auxilios, seguridad y protección en las diferentes marchas, en las asambleas; además proteje los sitios sagrados y realiza levantamiento de cadáveres. Realiza también funciones de control en los territorios, supervisando el ingreso a los resguardos con retenes. Los miembros de la Guardia Indígena no reciben sueldos, de modo que su actuar es voluntario. La Asamblea Mayor es el máximo órgano de decisión y juzgamiento de los diferentes pueblos que conforman el CRIC; en una gran reunión se toman decisiones basadas en los diferentes elementos probatorios, como son los dictámenes del INMLCF; dentro de dicho proceso quedan como soportes las actas de juzgamiento, las cuales incluyen las decisiones que toma este órgano colectivo y garantiza su cumplimiento y transparencia ${ }^{21}$.

\section{Derechos humanos y los pueblos indígenas del Cauca}

Los indigenas colombianos, en especial los que habitan en el departamento del Cauca, han sido víctimas de violaciones de derechos humanos, por lo tanto, son vulnerables y actualmente luchan por el reconocimiento de los derechos culturales civiles y políticos.

Las comunidades indígenas, campesinas y negras, sufren todas las consecuencias de los sucesos violentos de la guerra. A menudo se presentan casos de privaciones

18 Sauca, conversación.

19 José Albeiro Camayo (coordinador regional de la Guardia Indígena del CRIC), en conversación con el autor, Popayán, 21 de mayo de 2018.

20 Ibíd.

21 Sauca, conversación. 
arbitrarias de la vida y de la libertad, restricciones no razonables a la libertad de circulación, apropiación ilícita de bienes de consumo y uso desproporcionado del poder armado y de la fuerza que quedan en la impunidad ${ }^{22}$.

Para hablar de derechos humanos y los pueblos indígenas es necesario tener una perspectiva histórica que permita comprender la situación en su desarrollo y aportes al reconocimiento de estos pueblos. En cuanto a los derechos de los pueblos indígenas, las Naciones Unidas establece: "Los derechos de los pueblos indígenas conforme al derecho internacional han evolucionado partiendo del derecho internacional vigente, incluidos los tratados de derechos humanos, en función de las circunstancias en que se encontraban estos pueblos y de sus prioridades, como los derechos a sus tierras, territorios y recursos y a la libre determinación" 23 .

A pesar de que los procesos de violencia, discriminación, abuso y exterminio de los pueblos indígenas han sido una constante histórica, el reconocimiento de sus derechos en el marco de los organismos internacionales como las Naciones Unidas es un tema más bien reciente. De hecho, la "Declaración" apenas surge el 13 de septiembre de 2007 en el marco de la Resolución 217 A de la Asamblea General. Para el caso que nos convoca, se hará referencia únicamente al ya citado artículo 5 de la "Declaración". En tal sentido se podría afirmar que la CP ya había definido una postura importante frente a la necesidad de generar autonomía a los pueblos indígenas.

En el plano mundial existen tres mecanismos de las Naciones Unidas centrados en los pueblos indígenas e incluso anteriores a la "Declaración" de 2007:

- Foro Permanente de las Naciones Unidas para las Cuestiones Indígenas (UNPFII). Establecido el 28 de julio de 2000, es el órgano asesor del Consejo Económico y Social (ECOSOC), "con el mandato de examinar las cuestiones indígenas en el contexto de las atribuciones del ECOSOC relativas al desarrollo económico y social, la cultura, el medio ambiente, la educación, la salud y los derechos humanos" 24 .

- Mecanismo de Expertos sobre los Derechos de los Pueblos Indígenas (MEDPI). Creado mediante la resolución 6/36 de 2007 como un órgano subsidiario del Consejo de Derechos Humanos de las Naciones Unidas (CDH), el principal organismo intergubernamental de derechos humanos de la ONU.

- La Relatoría especial sobre los derechos de los pueblos indígenas, creada por la Comisión de Derechos Humanos en 2001, ha ejercido activamente sus mandatos y capacidades para promover el reconocimiento y ejercicio de los derechos de los pueblos indígenas, como se indica en la "Declaración".

22 Naciones Unidas, "Declaración".

23 Naciones Unidas, "Los pueblos indígenas y el sistema de derechos humanos de las Naciones Unidas", Folleto informativo n.o 9, Rev. 2, 2013, https://www.ohchr.org/Documents/Publications/fs9Rev.2_SP.pdf.

24 Naciones Unidas - Pueblos Indígenas, "Foro permanente", Departamento de asuntos económicos y sociales, acceso el 4 de junio de 2019, https://www.un.org/development/desa/indigenous-peoples-es/sesiones-del-foro-permanente.html. 

justicia ordinaria, un intercambio cultural posible

En un informe de Victoria Tauli-Corpuz, Relatora Especial sobre los derechos de los pueblos indígenas, se habla de que se está desencadenando una crisis mundial:

Si bien la vulnerabilidad de los pueblos indígenas a las agresiones mientras intentaban defender sus tierras ha sido una inquietud de larga data del mandato, en los últimos años ha sido motivo de grave preocupación el drástico aumento de esos actos. La Relatora Especial ha dejado constancia del importante aumento del número de esas agresiones en el Brasil, Colombia, el Ecuador, Filipinas, Guatemala [...] entre otros países. Esos mismos países han sido señalados por otros mecanismos de derechos humanos y organizaciones de la sociedad civil que detectan agresiones contra los pueblos indígenas y a este respecto han indicado en todo momento que esos países representaban situaciones especialmente preocupantes; se ha instado reiteradamente a las autoridades de tales países a que adopten medidas para mejorar la protección de los pueblos indígenas, pero no lo han hecho.

[...]

Si bien el asesinato de defensores indígenas representa la peor violación de los derechos humanos, esas agresiones suelen ocurrir en el contexto de violencia y amenazas contra ellos y sus comunidades, lo que entraña desapariciones forzadas, desalojos forzosos, acoso judicial, detenciones y encarcelamientos arbitrarios, limitaciones a la libertad de expresión y la libertad de reunión, estigmatización, sometimiento a vigilancia, prohibición de viajar y acoso sexual. ${ }^{25}$.

Lo anterior pone en evidencia el serio panorama de violación de los derechos humanos y las infracciones al derecho internacional humanitario a las que se ven sometidos los pueblos indígenas de varios países, entre ellos Colombia, donde, en medio del fuego cruzado, se han visto sometidos históricamente a una alta situación de vulnerabilidad. En una visita realizada a Colombia por el Foro Permanente de la ONU para las Cuestiones Indígenas en 2010, determinó que "La situación de los derechos humanos de los pueblos indígenas en Colombia continúa siendo sumamente grave, crítica y profundamente preocupante, a pesar del reconocimiento constitucional de estos derechos". Y tal como lo afirma la Relatora Especial, en la actualidad se hace más crítica la situación si se tiene en cuenta que existe un profundo desconocimiento sobre sus derechos económicos, sociales, y culturales. Se puede afirmar que la violencia y otros crímenes, así como el desplazamiento forzado y el confinamiento, amenazan la supervivencia física y cultural de los pueblos indígenas de Colombia.

Para abordar el tema de los derechos humanos de los pueblos indígenas del Cauca es necesario también recurrir a la conceptualización de lo que se entiende por resistencia. En el caso de los pueblos indígenas esta resistencia se encuentra asociada a diversas causas que van desde las mismas condiciones de segregación cultural hasta la reclamación de tierras de las cuales han sido desplazados.

25 Victoria Tauli-Corpuz, "Informe al Consejo de Derechos Humanos - 2018. Agresiones y criminalización a que se ven sometidos los pueblos indígenas que defienden sus derechos”, Asamblea General, Naciones Unidas, http://unsr.vtaulicorpuz.org/site/index.php/es/ documentos/informes-anuales/251-report-hrc2018. 
En tal caso afirman Rudqvist y Anrup: “Para el movimiento indígena, la resistencia civil es un ejercicio de autonomía y práctica comunitaria frente al Estado, los actores del conflicto armado y los intereses económicos transnacionales. [...] La resistencia incluye varios elementos como las estrategias de la participación comunitaria, la participación política, la educación y capacitación, el diálogo, el plan de emergencia, la Guardia Indígena y las asambleas permanentes" ${ }^{26}$. Sin duda, esta estrategia de resistencia indígena ha ocasionado un sinnúmero de enfrentamientos con el ejército nacional, y violaciones de los derechos humanos. En cuanto a la promulgación de Colombia como una nación multiétnica y pluricultural y el reconocimiento formal de la identidad específica y la herencia cultural de los pueblos indígenas colombianos previstos por la $\mathrm{CP}$, afirman los catedráticos Rudqvist y Anrup:

Las autoridades tradicionales, tales como cabildos y asociaciones indígenas, son reconocidas como entidades públicas de una naturaleza especial. Conservan su carácter de autoridades tradicionales, pero tienen además un mandato público de ejercer las funciones de administración y gobierno establecidas por la ley. La Constitución declara que cada comunidad o pueblo tiene el derecho de formar su propia forma de gobierno. La jurisdicción especial indígena constituye un ámbito de derecho propio que coexiste con la ordinaria y se reconoce no solo como derecho de costumbre que requiere reconocimiento, sino también como una especie de sistema de regulación o control practicado por cada pueblo indígena, que tiene la autonomía completa de ejercerlo dentro de su territorio. Además, por ser los indígenas sujetos colectivos de derechos, las vulneraciones individuales constituyen violaciones a los derechos del grupo ${ }^{27}$.

Según la Organización de las Naciones Unidas y el CRIC, el hecho de que las comunidades indígenas se encuentren en medio del conflicto armado colombiano está relacionado con los conflictos históricos de tierras que se vienen presentando desde hace varios siglos, fomentados por los encomenderos y los grandes latifundios y cultivos, sobre todo en el norte del Cauca y sur del Valle del Cauca, los cuales aún están en disputa; su recuperación hace parte de la lucha y resistencia actuales.

\section{Diálogo de saberes y construcción intercultural para la justicia indígena}

El título expuesto podría convertirse, seguramente, en una tesis muy amplia, que en todo caso necesitaría de un espacio más extenso que este; sin embargo, es necesario abordar algunos elementos de contenido filosófico y pedagógico que ayudarán a entender la perspectiva de lo que se podría entender como diálogo de saberes y construcción intercultural para la justicia indígena, en el marco del

\footnotetext{
26 Anders Rudqvist y Roland Anrup, "Resistencia comunitaria en Colombia. Los cabildos caucanos y su guardía indígena”, Papel Político 18, n.o 2 (2013): 524, acceso el 4 de junio de 2019, https://revistas.javeriana.edu.co/index.php/papelpol/article/view/7421.

27 Ibíd., 521.
} 
actual orden jurídico colombiano. Bajo estos preceptos será posible entender la importancia de formar discectores forenses indígenas desde la academia normalizada, que les permita a los pueblos indígenas del Cauca hacer "justicia propia" con evidencias concretas, sin romper sus esquemas de juzgamiento ancentrales.

En este contexto desde la mirada de los movimientos populares y sociales según lo afirma María Mercedes Palumbo la educación para los pueblos indígenas del Cauca es algo deseable y significa la concreción de un derecho históricamente negado, o la resignificación de una ausencia que ellos consideran necesario reencausar. ${ }^{28}$

Para iniciar, se llevarán a cabo algunas reflexiones conceptuales sobre interculturalidad y diálogo de saberes, partiendo en primera instancia de la propuesta de Paulo Freire vista por otros autores, y se ampliará de manera muy sintética a lo expuesto en Teoría de la acción comunicativa de Jürgen Habermas. Esto permitirá comprender, desde la práctica, las nuevas tendencias para hacer "justicia propia" en los pueblos indígenas del Cauca, aprovechando la posibilidad que les presenta tanto la "Declaración", como el citado artículo 5 sobre jurisdicciones especiales de la CP de Colombia.

Lo intercultural en el mundo globalizado es un tema que presenta aristas tan diversas, que, sin duda, no logran tocarse entre sí. Un referente filosófico importante para América Latina y particularmente para Colombia es el pedagogo y filósofo brasileño Paulo Freire. Si bien la idea de interculturalidad no estaba presente en sus conceptualizaciones, su metodología y práctica educativas eran un hecho intercultural, vale decir, una relación entre culturas diferentes. No obstante, no se trata únicamente de dicha relación, sino de todo lo que ello implica. Históricamente, se le ha imprimido una relación de poder: "Es decir, existe un carácter político ineludible en lo intercultural y esta politicidad se yuxtapone, en el contexto concreto de la práctica educativa intercultural, con la politicidad intrínseca de la educación" 29 .

Históricamente la relación de interculturalidad es una relación de sometimiento del conquistador con el colonizado, del que sabe y el que no: "El sometido, el oprimido, el colonizado, no sabe nada relevante y nada tiene para enseñar, debe someterse al poder y al saber del conquistador a riesgo de ser aniquilado" ${ }^{30}$. El que domina no aprende la lengua del dominado, etcétera. Paulo Freire, desafiando la filosofía colonizadora, propone una relación pedagógica crítica y dialógica: diálogo de saberes. Los sujetos no son tabula rasa. "La educación liberadora viene a reconocer que el alumno tiene saberes previos y que el que enseña no sabe todo, de esta forma el proceso de aprendizaje se vuelve un

28 Palumbo, María Mercedes. "Educación en movimientos populares rurales: un estado del arte". Revista Historia de la Educación Latinoamericana. Vol. 18 No. 26 (2016): 219-240. https://www.redalyc.org/pdf/869/86945261011.pdf

29 Jorge Alejandro Santos, "Filosofía intercultural y pedagogía. Releyendo a Paulo Freire" (Universidad de Buenos Aries, Facultad de Filosofía y Letras, 2015), acceso el 4 de junio de 2019, https://www.teseopress.com/tesisdoc.

30 Ibíd. 
acto de creación de conocimiento nuevo al que todos contribuyen enseñando y aprendiendo" ${ }^{31}$.

El diálogo de saberes o diálogo intercultural, como lo plantea el filósofo y académico argentino Jorge Alejandro Santos, es más un "proyecto" que una realidad. Esto se manifiesta claramente en las prácticas pedagógicas y académicas de nuestro medio, donde perviven prácticas autoritarias y sometedoras. "Sin embargo, conocimiento debe entenderse no como un simple 'tomar nota'. Pues se trata de un proceso profundo de información en el que nos (in)formamos y nos dejamos formar (en el sentido de dar forma) por lo que conocemos (la otra cultura)." ${ }^{\prime 32}$.

Pero no bastan las buenas intenciones de posicionarse en una relación de saberes interculturales, en un diálogo de culturas profundamente diversas, como ocurre entre el pensamiento occidentalizado y la cultura de los pueblos indígenas, cuyas cosmovisiones son completamente opuestas. Es allí donde Habermas nos coloca en situación crítica, dado que existe una hegemonía del primero sobre el segundo.

En este caso, ¿cuándo se habla de una relación dialógica? Es decir, en el diálogo de saberes podríamos analizar el papel de la interpretación de los sujetos de interacción en un contexto de no subordinación de saberes. Es aquí donde el concepto de acción comunicativa tiene sentido

[...] el concepto de acción Comunicativa se refiere a la interacción de por lo menos dos sujetos capaces de lenguaje y de acción (ya sea con medios verbales o con medios extraverbales) entablan una relación interpersonal. Los actores buscan entenderse sobre una situación de acción para poder así coordinar de común acuerdo sus planes de acción y con ello sus acciones. El concepto aquí central, el de interpretación, se refiere primordialmente a la negociación de definiciones de la situación suceptible de consenso. En este modelo de acción el lenguaje ocupa [...] un puesto predominante ${ }^{33}$

Es así como en la CP y la "Declaración" se promueve una acción comunicativa fundada en el concepto de acción teleológica, "que presupone relaciones entre un actor y un mundo de estados de cosas existentes" 34 . En el caso de la relación dialógica entre las acciones culturales de los pueblos indígenas en sus prácticas de "justicia propia" y los supuestos de verdad que definen las prácticas forenses utilizadas en el modelo académico y del INMLCF que busca también hacer justicia en el campo del juzgamiento ordinario, propio de un pensamiento occidentalizado, nos encontramos con dos mundos que requieren ser interpretados, hacia la búsqueda de un mismo fin.

\footnotetext{
31 Ibíd.

32 Ibíd.

33 Jürgen Habermas, Teoría de la acción comunicativa (Madrid: Taurus, 1999), 124.

34 Ibíd., 125.
} 
Habermas también, lo expone en su teoría de la acción comunicativa cuando establece que "un mundo social consta de un contexto normativo que fija qué interacciones pertenecen a la totalidad de relaciones interpersonales legítimas. $\mathrm{Y}$ todos los actores para quienes rigen las correspondientes normas (por quienes son aceptadas como válidas) pertenecen al mismo mundo social"35. Por lo tanto, la comprensión de las acciones, tanto de unos como de otros, indefectiblemente debe llevar a un diálogo intercultural, en función de la construcción de un conocimiento orientado a un mismo fin: la justicia.

Es así como se establecieron acciones de interacción cultural para la formación de disectores indígenas del Cauca como una contribución desde la académica para el ejercicio de una "justicia propia", en la perspectiva de la aplicación de los derechos fundamentales universales de las personas. Una experiencia exitosa que nos proponemos dar a conocer por los aportes que tanto la academia pudo comprender en cuanto a las prácticas mismas del pueblo indígena, como por reciprocidad que estos pueblos tuvieron con el objetivo de querer mejorar sus prácticas dentro de sus comunidades y sus procesos de juzgamiento.

A partir del año 2016, debido a las múltiples críticas por parte de la justicia ordinaria en cuanto a las condenas y juzgamientos realizados por la justicia indígena en diversos delitos o desarmonizaciones, aunado a las reclamaciones hechas por la defensa en las mismas asambleas mayores, las autoridades indígenas del Cauca, en busca de obtener elementos objetivos y técnico-científicos, no debatibles fácilmente desde la retórica de los médicos tradicionales y de la misma defensa, solicita al INMLCF la realización de autopsias médico-legales, dictámenes de violencia sexual y pruebas genéticas de paternidad, entre otros exámenes, como argumentos científicos probatorios.

Esta decisión de la autoridad indígena de apoyarse en instituciones del Estado, demuestra la flexibilidad de la "justicia propia" en búsqueda de la verdad y el mejoramiento de sus procesos. Es una decisión que promueve el acercamiento por parte del INMLCF y la Universidad del Cauca, con el propósito de establecer un diálogo de saberes que permitirá intercambiar experiencias y conocimientos como mediación para fortalecer la justicia en general.

A partir de esta decisión, se inicia un proceso de diseño de contenidos y necesidades para la capacitación en procedimientos técnicos forenses, consensuado entre las autoridades indígenas y las instituciones intervinientes. Estos acuerdos se lograron mediante una rigurosa interacción, acercamiento, comprensión y diálogo permanente entre los participantes.

En esta interacción entre el CRIC y la universidad surgió, por parte de los pueblos indígenas, la propuesta de construir un curso teórico-práctico de disector asistente en procedimientos forenses, el cual fue aprobado por el consejo de la Facultad de Ciencias de la Salud de la Universidad del Cauca. Se diseñó un curso corto, con una duración de tres meses, y se desarrolló con el aval del

35 Ibíd., 127. 
programa de Medicina de dicha universidad, el centro de educación virtual y a distancia y como escenario de práctica, el INMLCF, seccional Cauca.

El curso se impartió en cinco módulos, cada uno de dos semanas, en temáticas de interés de la mujer indígena seleccionada por su perfil, por parte de la comunidad del resguardo y del CRIC. Las temáticas fueron las siguientes: lofoscopía, toxicología, evidencia traza, residuos de disparo, biología, antropología, clínica, odontología, patología, gestión documental, gestión ambiental, gestión de salud y seguridad en el trabajo (técnicas de asepsia y antisepsia, elementos de protección personal), servicio al cliente, sistema penal acusatorio, legislación laboral, manejo de paquete ofimático, manejo de correo electrónico, manejo de motores de búsqueda, manejo de equipos informáticos, seguridad informática y calidad del dato, anatomía humana, disecciones generales, recolección y manejo de muestras, documentación de hallazgos, tipos de necropsia y procedimientos forenses, técnicas especiales de disección de cadáveres, toma y manejo de muestras para histopatología.

\section{La formación de disectores y asistentes forenses en Colombia}

Este tipo de formación se ha caracterizado por ser práctica, técnica e instrumental, basada en la observación, la praxis y la adquisición de conocimientos empíricos; sin embargo, para la formación de indígenas, dicho paradigma cambia por una estrategia de libertad de aprendizaje, basado en la autonomía y la autoformación.

El curso se desarrolló en su totalidad en las instalaciones del Instituto Nacional de Medicina Legal, en la morgue y en el área de clínica y patología. Se realizaron prácticas y lecturas básicas de los temas, pero en esencia fue noventa por ciento práctico y diez por ciento teórico, con lecturas dirigidas. Se realizó una evaluación cada semana con un breve examen escrito de desempeño y un examen práctico sobre técnicas de disección y manejo de evidencias a cargo del perito-tutor asignado en el marco del convenio suscrito entre las instituciones participantes.

Este cambio pedagógico se debe a la resistencia que existe en la cosmovisión de los pueblos indígenas, a consecuencia de la desconfianza motivada por los incumplimientos históricos del Estado. Por esta razón la IAP muestra sus fortalezas en el sentido de que los acuerdos y compromisos planteados por las comunidades y las entidades generaron puentes de comunicación, entendimiento, confianza y flexibilidad en un intercambio y respeto por las diferentes creencias y cosmovisiones. De acuerdo con el testimonio de Liset Natalia Murillo, la primera mujer participante del programa, "Se puede interpretar que mi formación es fundamental para el interés que tiene mi comunidad en la aplicación de estos conocimientos en función del servicio para la comunidad, el cabildo y el pueblo". Este argumento describe claramente la armonización, la manera como ella puede contribuir al proceso de juzgamiento en virtud de hacer "justicia". 
Para mí es una satisfacción muy grande saber que, al ser la primera persona indígena que decide capacitarse en esta área, puede abrir puertas a los demás jóvenes de mi comunidad para que obtengan muchos más conocimientos acerca de esto y que tengan más oportunidades laborales, además que nos brindan la posibilidad de aportar muchas ideas a nuestro resguardo. ${ }^{36}$

\section{Nuevos conocimientos aprendidos por las instituciones estatales y la jus- ticia tradicional}

La Universidad del Cauca, con el apoyo y guía de los pueblos indígenas, en un diálogo de saberes, diseñó un modelo pedagógico y curricular que se ajustó a las necesidades de los indígenas para el fortalecimiento del constructo de justicia intercultural, mediante un curso que se impartió en el año 2018 durante los meses de agosto a diciembre, se logró desarrollar y abrir puertas para los integrantes de las comunidades con el fin de que desarrollasen habilidades, capacidades y competencias en apoyo a la "justicia propia". Desde esta perspectiva se lograron describir los aprendizajes y experiencias de los pueblos indígenas frente al trabajo técnico-científico del INMLCF y la universidad, en un intercambio de conceptos, saberes, conocimientos y cosmovisión, los cuales son altamente valorados por todos los intervinientes desde lo técnico-científico y lo cultural.

Este proceso requiere de tiempo y maduración debido a la cosmovisión y cultura de los pueblos con los que se ha iniciado este trabajo. La desconfianza es justificada, debe ser comprendida y deben desaparecer la hegemonía institucional para que surjan culturas sensibles y humildes que permeen procesos de intercambio cultural igualitarios, enriqueciendo a la sociedad.

Es necesario que la sociedad y la justicia ordinaria se apropie y reconozca términos como armonización, desarmonización, cateo, guardia indígena, comunidad, asamblea, comunero, entre otros, que hacen parte del proceso de justicia propia y que permitiría que el perito se aproxime y aporte desde lo técnico a una transformación del lenguaje, para un mayor entendimiento y contextualización, dentro del denominado enfoque diferencial, hacia una justicia intercultural.

La investigación criminal en los territorios indígenas estaba basada en las prácticas de los the'walas o médicos tradicionales, quienes realizaban cateos o pesquisas, en algunas ocasiones acertadas; sin embargo, estas prácticas culturales ancestrales se han transformado debido al trabajo de apoyo de INMLCF y la Universidad del Cauca, el cual ha sido considerado como muy importante y valorado.

El enfoque diferencial en la investigación hace parte de los procesos. Los aportes técnico-científicos son llevados a los juicios en delitos que ocurren dentro de los territorios indígenas, gracias a los cuales la justicia indígena ha sido más ágil

36 Liset Natalia Murillo, testimonio. 
en la aplicación de justicia propia. En comparación con la justicia ordinaria, los juicios pueden durar pocas semanas en la justicia propia.

Esto que está ocurriendo es el resultado de un proceso de la lucha, de la posición política y de empoderar a la justicia indígena para que ellos puedan aplicar con transparencia la justicia propia y sea posible hacer un trabajo articulado.

\section{La justicia indígena y sus procedimientos}

La justicia indígena ha desarrollado tradicional y oralmente la aplicación de las sentencias. Con el paso del tiempo, los actores y los delitos cambian y se presentan nuevos casos que requieren de nuevos insumos como aporte desde la jurisdicción ordinaria; por mencionar un ejemplo, en la justicia indígena hay condenas de cuarenta años sin ningún tipo de beneficio.

En relación con lo afirmado por Fernando Caso Chate y Joe Sauca, en comparación con la justicia ordinaria, en la que los colombianos procesados judicialmente tienen rebajas de penas, las comunidades indígenas se preguntan "¿qué se está haciendo ahora a través de la transformación de los conceptos y grados de delitos?, ¿dónde se plantean, por ejemplo, las condenas diferenciales?". Dichas preguntas sugieren que la aplicación de penas ya está cambiando desde lo humano, trasladando y re-integrando a los comuneros a sus comunidades, dándoles otra oportunidad en cárceles o sitios de re-armonización rurales.

Existe la necesidad de capacitar a la Guardia Indígena para atender algunos casos lejanos dentro de los territorios. Ya que es un brazo fuerte de la autoridad, la Guardia Indígena requiere tener conocimiento sobre cadenas de custodia, embalaje de evidencias, manejo de la escena, conocimiento de manejo de escena en homicidio y recolectar elementos en la escena, así como otros procedimientos técnicos. El curso diseñado por la Universidad del Cauca y el programa de Medicina es un avance en este sentido.

José Albeiro Camayo Coordinador Regional de la Guardia Indígena explica: La guardia atiende diferentes tipos de delitos, que ellos denominan desarmonización, ocurridos en sus diferentes territorios, estos pueden ser asesinatos, secuestros y violaciones, siendo estos los más graves, y otros delitos considerados más manejables, como los robos, entre otros. Frente a los delitos, la justicia indígena desarrolla juicios comunitarios en los cuales intervienen la autoridad y la contraparte, que es el acusado, su familia, personas amigas de la familia o del acusado, de la misma comunidad. Actualmente es más complicado llevar a cabo el juicio, puesto que se han generado diferencias en las decisiones, como las penas consideradas muy bajas. En esa medida, el sustento técnico-científico ha servido para establecer la culpabilidad, que ya no se basa únicamente en el cateo con el mayor (sea autoridad o médico tradicional) sino que además, tiene en cuenta el aporte de elementos materia de prueba, como por ejemplo las pruebas genéticas y los resultados de autopsias; estas pruebas han cambiado considerablemente esta dinámica de juzgamiento. 
En el proceso de juzgamiento son importantes la coordinación y el trabajo conjunto entre el INMLCF, quienes producen el informe, y las orientaciones de los mayores, que pueden ser desde cinco hasta doce personas, dependiendo de la complejidad de la situación, por ejemplo si alguien aparece muerto y no se sabe nada. En estos casos primero se basa en la sospecha, los médicos inician la investigación y ellos las estudian y mandan a capturar al sospechoso, a partir de ahí es la asamblea la que decide.

Según lo anotado por Joe Sauca Dentro de la comunidad también hay defensores que en la asamblea son llamados a apoyar por parte de la familia del acusado o los vecinos; hay discusiones entre la comunidad y le asignan la pena al acusado en la asamblea. A esta asisten aproximadamente tres mil comuneros para tomar la decisión, el abogado puede apelar la decisión y en estos casos los informes son muy importantes porque dan validez o fortaleza a la decisión de la asamblea

Según Joe Sauca La autoridad indígena coordina con la guardia indígena y es finalmente la comunidad en la asamblea mayor quien decide la aplicación de justicia propia, se siguen conductos regulares y procedimientos que permiten un juicio coordinado, transparente y ágil para evitar la cultura del odio y venganza, es importante lograr la reconciliación entre víctima y victimario, teniendo en cuenta que este proceso es histórico y las comunidades indígenas ancestralmente lo han venido haciendo.

De acuerdo con Fernando Caso Chate, José Albeiro Camayo, Joe Sauca y Alex Basto $^{37}$ en el grupo focal y de entrevistas el fuete tiene como propósito la re-armonización, rectificar el camino de la persona con ayuda del médico tradicional y así lograr la armonización, situándose frente a la comunidad, reconociendo sus errores y reubicando su camino. Para la sociedad occidentalizada, esto puede ser considerado como tortura, pero desde la cosmovisión indígena es legal y logra la corrección de los errores por parte de los infractores.

Así lo afirman las autoridades Liset Natalia Murillo y Joe Sauca:

La gente cambia, se vuelve trabajadora, responsable y se portan en la comunidad bien, y después del arrepentimiento han sido grandes personas que le han ayudado a su comunidad obviamente con delitos menores, hay por ejemplo el comunero que se robó una gallina o entró en una casa, pero estos compañeros se pueden rehabilitar y llegan a fortalecer la Guardia Indígena. La armonización es hacer que la persona piense y reconsidere lo que hizo.

Existe un conflicto entre los jueces y la justicia indígena debido a que no hay reconocimiento por parte de los jueces de sus procesos, ni tampoco de la toma de decisiones; ellos defienden que solo juzgan a quienes han cometido delitos en sus territorios y a los miembros de su comunidad.

Para explicar en qué consiste la desarmonización, algunos entrevistados como Sauca, Murillo y Camayo, afirman: "Sea dentro del territorio sea indígena

37 Alex Basto (auxiliar jurídico en derechos humanos del CRIC), en conversación con el autor, Popayán, 21 de mayo de 2018. 
o no, muchas veces las mismas personas que desarmonizan se acogen a la jurisdicción indígena, los indígenas que desarmonizan merecen otra oportunidad a través de la rearmonización en centros de armonización, que la persona regrese, que haya perdón y se reincorpore a la comunidad y no se desarraigue de su cultura, proponen fincas integrales productivas".

Según Fernando Caso Chate:

La armonización es una forma de hacer que la persona piense y reconsidere lo que hizo. Les da la posibilidad de reembarcar su senda, renovar su camino, no los marca para toda la vida; la concepción indígena y de autoridad propia es la forma de pensar sabiamente qué es lo que quiere, la desarmonización es el delito la armonización es la corrección, la comunidad es el juez de la rehabilitación, el ejercicio de la comunidad es que todos son jueces y esta vigilancia es por años que lo están mirando, la gente lo revisa y ve que lo armonizaron, todos en el territorio nos conocemos y sabemos cuál es el comportamiento y el cambio de la persona.

Si la persona continúa delinquiendo, se les sigue armonizando, existen varias medidas para delitos menores, calabozo, cepo, fuete, y si es inmanejable, existe el destierro y se va de la comunidad y la misma comunidad lo decide.

El cateo es parte de la medicina tradicional y es la forma mediante la cual el médico tradicional o the'wala, cultiva los dones. No todos los médicos son espirituales y son varios dones que existen, el que pulsea, el espiritual y el que trabaja con plantas $y$ de acuerdo a la creencia y cosmovisión de cada pueblo. Este procedimiento es muy preciso, y se le dice al acusado del delito, "ven te paso la mano", y con solo tocarlo le dice "estás asustado", dentro del cateo, y uno lo interroga alrededor de lo que cometió la persona, qué error cometió, y cómo lo hizo, eso se llama espiritualidad, estar arriba y abajo... asi determinamos que es culpable [...] entonces los médicos espirituales con su trabajo llevan a esa persona y espiritualmente ve cómo está su situación y ve qué culpabilidad hay, esto es místico y es muy difícil de mirar pero que es una realidad y nos ha permitido resolver múltiples situaciones mediante esta [el cateo], y es parte de nuestra cosmovisión, son unas marcas en el cuerpo, el médico siente las señas en el cuerpo y hay casos en que el médico ha acertado y la persona reconoce su culpa.

Este método de investigacion mágico, religioso, místico y espiritual, denominado "cateo", es un elemento muy importante para algunas comunidades, puesto que es el médico tradicional quien lleva su dictamen a la asamblea del resguardo a través de su trabajo espiritual, según lo afirma la autoridad. Fernando Caso Chate asegura: "Se sabe que es la persona culpable y la asamblea decide la amonestación o corrección".

De acuerdo con Joe Sauca, es así que la asamblea, como máxima autoridad, es convocada por la misma "gente" o comunidad del cabildo; una vez anunciada, al poco tiempo comienzan a llegar todos los habitantes del resguardo. Cabe anotar que la figura de cabildo no existe en sus concepciones y cosmovisión. Liset Murillo y Joe Sauca niegan esta figura, la consideran una idea administrativa impuesta y oligárquica, pero sirve para contextualizar su territorio y las comunidades que allí viven, y aunque el término genera resistencias, es utilizado como 
mecanismo de direccionamiento del resguardo, de manera que las decisiones son tomadas en conjunto y no son el gobernador o la autoridad indígena quienes tienen el poder absoluto, ya que el poder es comunitario, democrático o colectivo.

Los integrantes del cabildo son funcionarios al servicio de la colectividad, sin poder ni salario, es decir, ocupan cargos de tipo administrativo que permiten una interacción con la sociedad occidental. El señor Joe Sauca afirma:

\begin{abstract}
Existe una ética muy estricta, frente a la comunidad y ella decide quiénes quieren que nos dirijan y nos representen, si alguien comete un error o se equivoca y la misma comunidad deja que pase su periodo y nunca lo vuelven a llamar y esa es la sanción y hasta allí llegó y la comunidad lo deja de lado. Este es un aspecto importante y fundamental que ha sido para estos pueblos una garantía para su supervivencia porque la ética y el manejo transparente de los recursos la corrupción casi nula o muy escasa garantiza la permanencia de los pueblos.
\end{abstract}

Tambien Camayo, Sauca y Caso Chate se refieren al tema de la Guardia Indígena y las autoridades, expresando que las sanciones morales y el rechazo o falta de credibilidad de las comunidades es la peor sanción moral: "Eso es lo más duro que nos pueda pasar en un territorio. La misma comunidad no lo deja avanzar. El periodo de cargo gobernador es de un año, algunos lo ampliaron a dos años para las consejerías. Se hacen además cambios de generaciones que se renuevan. Si la persona no lo hizo bien no la vuelven a nombrar y la aíslan socialmente"

Según Sauca, también existen "los mandatos":

[Son] las máximas decisiones que toman los pueblos y son de orden obligatorio y también son la plataforma estratégica, política y jurisdiccional para la justicia indígena. Son el producto de asambleas en donde la comunidad los expide y quedan expresados en actas, son difundidos y esto obliga al CRIC y a la autoridad a velar por su cumplimiento y la Guardia Indígena quien velara, alertara y guardara su acatamiento inapelable y estricto por todos los habitantes de los territorios, esto en razón que existen mestizos casados con indígenas quienes por habitar en los territorios deben someterse a estos mandatos.

Es importante partir desde el punto de vista humanístico y cultural, que el respeto por las costumbres y la cosmovisión de los pueblos indígenas debe ser la carta de navegación para todos aquellos que planteen proyectos similares de tipo cualitativo.

En palabras de Caso Chate y Sauca, el término “justicia propia” nos enseña muchos elementos que mezclan la cultura, las costumbres, la familia, la comunidad, y en los términos desarmonización y armonización, que para la comunidad no indígena debería ser tomado como un ejemplo de análisis y asimilación. Además, la ética y el compromiso para y con "la comunidad" son fundamentales como aprendizaje para la sociedad mestiza o "hermanos menores". 


\section{Una sucinta historia de vida}

Liset Natalia Murillo nació el 11 de junio de 2000 en la ciudad de Popayán, en una familia conformada por su madre, Yuly Andrea Murillo, quien actualmente es ama de casa, su padre, Mario Fernando Maca, coordinador del programa de educación del CRIC, y su hermana menor, Shirley Maca Murillo. Toda su niñez la pasó en una zona rural rodeada de naturaleza y animales debido a la gran influencia de sus padres por su amor al campo. En el departamento del Cauca las comunidades indígenas son rurales, la crianza en la infancia generalmente está a cargo de la madre y el padre es un proveedor, los pueblos indígenas desde su cosmovisión educan a sus niños con gran compromiso por su tierra y por sus tradiciones, además por sus lenguas, con una tradición oral que se transmite de una generación a otra.

Liset Nathalia estudió en un colegio femenino dirigido por religiosas, como parte de la cultura conservadora y religiosa de la ciudad de Popayán, su adolescencia estuvo muy marcada por diferentes cambios, entre ellos la separación de sus padres debida a múltiples diferencias entre ellos, por lo que tuvo que, en su papel de hermana mayor, tuvo que hacer de ama de casa y ayudar no solo en las labores de la casa sino en el cuidado de su hermana mientras su padre trabajaba.

A pesar de la resistencia histórica de los pueblos, es evidente la influencia de la occidentalización de la educación en la formación básica y media, la imposición del idioma, la escritura, con una formación moral y civica en las ciudades, donde se observa la hegemonía de las estrategias curriculares y pedagógicas imperantes en el país, de las cuales los pueblos indígenas no consiguen abstraerse a pesar de las diferentes estrategias de resistencia y rebeldía, como, por ejemplo, crear las escuelas indígenas en los territorios, idea promovida por Quintin Lame $^{38}$.

Al finalizar sus estudios de bachillerato, Liset Natalia comenzó a cuestionarse sobre la dirección que debía darle a su vida y decidió buscar opciones para estudiar de acuerdo con sus capacidades y gustos; concluyó que le atraían mucho los asuntos militares y judiciales, así que optó por hacer curso de técnico sabatino en investigación judicial y criminal, proceso durante el cual desarrolló diferentes intereses que eran muy significativos para su proyecto de vida. En la actualidad las posibilidades de acceso a la educación superior por parte de la población indígena son escasas o inexistentes. Si bien la Universidad del Cauca oferta cupos especiales a poblaciones indígenas, deben cumplir con los requisitos de admisión, lo cual genera frustraciones muy fuertes entre estas minorías.

Por intermedio del CRIC y del proyecto de investigación-acción es elegida como la persona más capacitada para el curso de disector forense y es escogida por su colectividad para asistir al Instituto Nacional de Medicina Legal y Ciencias Forenses con la ayuda del CRIC como disectora con el fin de apoyar la

38 Francisco Romero Loaiza, "Manuel Quintin Lame: sabiduría y saber escolar", acceso el 25 de marzo de 2019, http://www.humanas. unal.edu.co/colantropos/files/5214/5615/3567/manuel_quintin_lame_sabiduria_y_saber_escolar.pdf. 
jurisdicción especial indígena gracias a su pertenencia al resguardo indígena de Poblazón. Liset Natalia expresa:

\begin{abstract}
Además de ser capacitada, la idea es aportar conocimiento en esta área a mi comunidad ya que no se tengo comprensión con exactitud de los procesos necesarios y obligatorios para manejar evidencias y el manejo de cadáveres en una situación delictiva o criminal.

La importancia de la capacitación es también abrir puertas a los integrantes de la comunidad que tengan habilidades, capacidades y osadía para hacer este tipo de procedimientos de manera que aporten en el esclarecimiento de eventuales hechos delictivos y claramente para aportar elementos contundentes que nos lleven a la plena aplicación de la justicia indígena.

La justicia indígena es un tema complejo, por eso uno de los objetivos es aportar a la armonización del territorio y que en procesos de acciones criminales nuestra comunidad tenga el conocimiento necesario para aplicar las sanciones pertinentes a cada situación, si es requerido, y donde los mismos integrantes de la comunidad, pero ya capacitados en el área forense, sean quienes argumenten y determinen la causa y la manera de los acontecimientos para que así la ejecución de la sanción sea mucho más contundente en este tipo de asuntos.
\end{abstract}

\title{
CONCLUSIÓN
}

La presente investigación-acción participativa logró que los pueblos indígenas del Cauca y las comunidades de los diferentes resguardos, en conjunto con el CRIC, el Instituto Nacional de Medicina Legal y Ciencias Forenses y la Universidad del Cauca, pudieran generar nuevas ideas para dar solución a las problemáticas en la aplicación de "justicia propia", educando a nuevos líderes indígenas $\mathrm{y}$, en este caso, la formación de la primera mujer indígena disectora forense del Cauca, descubriendo nuevas potencialidades para las mujeres como líderes de procesos.

Esta investigación-acción generó la transformación social dentro de los pueblos indígenas en cuanto a la credibilidad de las pruebas objetivas forenses y la introducción del conocimiento técnico-científico en la aplicación de la "justicia propia", transformando y apoyando a las evidencias propias, mágicas y culturales, mediante la ciencia forense actual.

Esta investigación-acción ayudó a la comunidad indígena y a los pueblos que la conforman a priorizar y diseñar, según sus necesidades reales, un curso para disectores exclusivo para los pueblos indígenas del Cauca, con el fin de dar solución a la problemática de las evidencias objetivas y pruebas técnico-científicas, generando conciencia de la necesidad de apoyo en los conceptos y dictámenes técnicos-científicos en los procesos llevados por la justicia especial indígena, lo cual modificó el entorno en el sentido de presentar las pruebas en las asambleas como elemento fundamental e indispensable de debate jurídico y procedimental 
para el juzgamiento y toma de las decisiones finales en los delitos que esta justicia administra en sus territorios.

\section{REFERENCIAS}

Arbeláez de Tobón, Lucía. “La Jurisdicción Especial Indígena en Colombia y los mecanismos de coordinación con el Sistema Judicial Nacional". República de Colombia, Rama Judicial del Poder Público. Guatemala: Consejo Superior de la Judicatura, 2004. Acceso el 4 de junio de 2019. https://www.cejamericas.org/Documentos/DocumentosIDRC/21LucaArbelaez.pdf.

Departamento Administrativo Nacional de Estadística - DANE. Acceso el 25 de marzo de 2019. https://www.dane.gov.co/.

"Encuentros y desencuentros entre la Justicia propia y la justicia ordinaria". Consejo Regional Indígena del Cauca - CRIC. 2018. Acceso el 7 de junio de 2019. https://www.cric-colombia.org/ portal/encuentros-desencuentros-la-justicia-la-justicia-ordinaria/.

Fals Borda, Orlando, Carlos Rodrigues Brandão. Investigación participativa. Montevideo: Instituto del Hombre /Ediciones de la Banda Oriental, 1986.

Flórez Schneider, Gloria. "Cuenten con nosotros para la Paz y NO para la guerra". Parlamento Andino. Acceso el 4 de junio de 2019. http://www.oidhaco.org/uploaded/content/article/1499238702.pdf.

Gómez Valencia, Herinaldy. “Justicias indígenas de Colombia: reflexiones para un debate cultural, jurídico y político. Pueblos Kogui, Arhuaco, Wiwa, Kankuamo, Nasa, Misak, Yanacona y Camëntśá". Centro de Documentación Judicial, 2015. https://www.ramajudicial.gov.co/ documents/4263275/0/LibroJusticiasIndigenas.pdf/e932af27-6ca8-462a-8f79-73cd14168426.

Habermas, Jürgen. Teoría de la acción comunicativa. Madrid: Taurus, 1999.

Naciones Unidas. "Declaración de las Naciones Unidas sobre los derechos de los pueblos indígenas". Acceso el 25 de marzo de 2019. https://www.un.org/development/desa/indigenous-peoples-es/declaracion-sobre-los-derechos-de-los-pueblos-indigenas.html.

Naciones Unidas - Pueblos Indígenas. "Foro Permanente". Departamento de Asuntos Económicos. Acceso el 4 de junio de 2019. https://www.un.org/development/desa/indigenous-peoples-es/sesiones-del-foro-permanente.html.

Quintín Lame, Manuel. "El derecho de la mujer indígena en Colombia". En Documentos para la historia del movimiento indígena colombiano contemporáneo, compiladores Enrique Sánchez y Hernán Molina, 32. Bogotá: MinCultura, 2010.

Rama Judicial de la República de Colombia. Comisión Nacional de Coordinación del Sistema Judicial Nacional y la Jurisdicción especial Indígena-COCOIN. Acceso el 25 de marzo de 2019. https://www.ramajudicial.gov.co/web/jurisdiccion-especial-indigena-y-el-sistema-judicial-nacio nal/acuerdos-y-compromisos.

Romero Loaiza, Francisco. "Manuel Quintín Lame: sabiduría y saber escolar". Acceso el 25 de marzo de 2019. http://www.humanas.unal.edu.co/colantropos/files/5214/5615/3567/manuel_quintin_lame_sabiduria_y_saber_escolar.pdf.

Rudqvist, Anders y Roland Anrup. "Resistencia comunitaria en Colombia. Los cabildos caucanos y su guardía indígena". Papel Político 18, n. ${ }^{\circ}$ 2, (2013): 524. Acceso el 4 de junio de 2019. https:// revistas.javeriana.edu.co/index.php/papelpol/article/view/7421.

Santos, Jorge Alejandro. "Filosofía intercultural y pedagogía. Releyendo a Paulo Freire". Universidad de Buenos Aires. Facultad de Filosofía y Letras. Acceso el 4 de junio de 2019. https:// www.teseopress.com/tesisdoc.

Semper, Frank. "Los derechos de los pueblos indígenas de Colombia en la jurisprudencia de la Corte Constitucional". Anuario del Derecho Constitucional Latinoamericano (2006): 764. http:// www.corteidh.or.cr/tablas/R21731.pdf.

Tauli-Corpuz, Victoria. “Informe al Consejo de Derechos Humanos - 2018. Agresiones y criminalización a que se ven sometidos los pueblos indígenas que defienden sus derechos". Asamblea General. Naciones Unidas, http://unsr.vtaulicorpuz.org/site/index.php/es/documentos/ 

justicia ordinaria, un intercambio cultural posible

informes-anuales/251-report-hrc2018.

Palumbo, María Mercedes. "Educación en movimientos populares rurales: un estado del arte". Revista Historia de la Educación Latinoamericana. Vol. 18 No. 26 (2016): 219-240. https:// www.redalyc.org/pdf/869/86945261011.pdf

https://doi.org/10.19053/01227238.4373

\section{Cómo citar:}

Álvarez Soler, Jaime Antonio. “Diálogo de saberes: justicia indígena del Cauca y justicia ordinaria, un intercambio cultural posible". Revista Historia de la Educación Latinoamericana. Vol. 21

No. 33 (2019): 35-59

https:https://doi.org/10.19053/01227238.9097

(c) (i) @) Esta obra está bajo una licencia Creative Commons. Reconocimiento-No Comercial-Sin Obra Derivada 2.5 Colombia. 


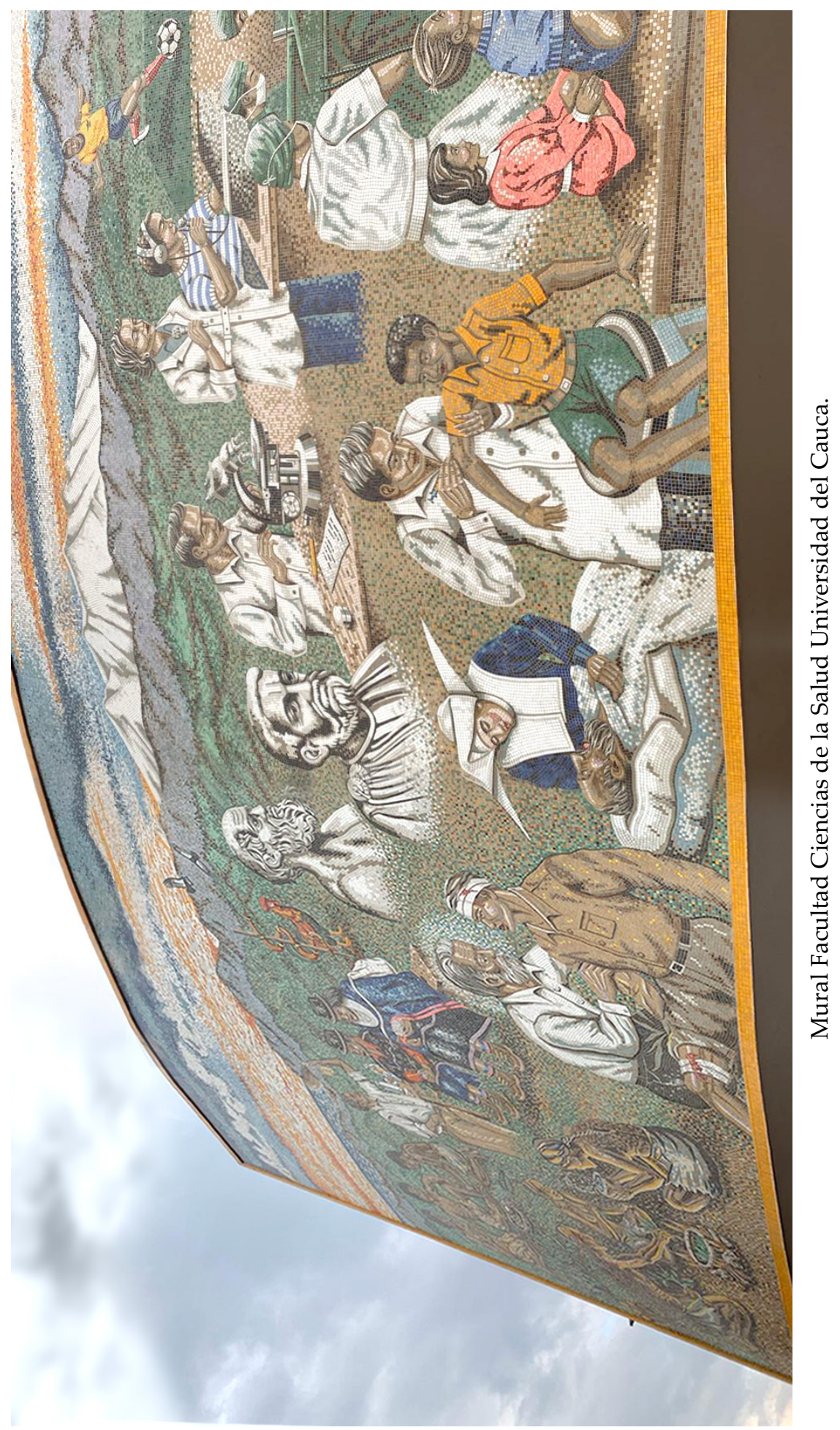

